\title{
POSSIBILITIES IN RELATIVISTIC THERMODYNAMICS FOR IRREVERSIBLE PROCESSES WITHOUT EXHAUSTION OF FREE ENERGY
}

\author{
By Richard C. TOLMaN \\ California Institute of Technology, Pasadena, California
}

(Received November 13, 1931)

Abstract

Previous applications of relativistic thermodynamics have been either to systems in static thermodynamic equilibrium or to non-static systems in which reversible processes are taking place. The present article deals with non-static systems in which irreversible processes take place. It is shown that the failure of the principle of energy conservation to hold in relativistic mechanics in its older simple form removes a classical limitation on the irreversible increases in entropy which can take place in an isolated system. The removal of this limitation provides possibilities in relativistic thermodynamics for irreversible processes to take place in an isolated system without ever reaching an unsurpassable state of maximum entropy and minimum free energy where further change would be impossible. Such possibilities are found to be illustrated by non-static models of the universe which could undergo a continued series of irreversible expansions and contractions without ever arriving at a state of rest. Special attention is given to a non-static model of the universe in which the irreversible annihilation of matter would take place in the later stages of expansion and the irreversible formation of matter out of radiation in the later stages of contraction. Finally, remarks are made concerning the bearing of the findings on the interpretation of phenomena in the actual universe.

\section{\$1. INTRODUCTION}

IN PREVIOUS articles the principles for an extension of thermodynamics to general relativity have been presented, ${ }^{1}$ and applications of the principles to several different problems have also been given. ${ }^{2}$

The new principles were first applied to problems in static equilibrium. For example in the case of a static gravitational field it was shown that the conditions for thermal equilibrium would necessitate a definite temperature gradient in order to prevent the flow of heat from regions of higher to those of lower gravitational potential. ${ }^{3}$ And in the case of a static Einstein universe, it was shown that the concentration of matter in equilibrium with radiation, assuming their interconvertibility, would be the same as in flat space-time. ${ }^{4}$

The principles were then applied to non-static systems in which reversible processes take place. In contradiction to a familiar conclusion of classical thermodynamics, the possibility was found in relativistic thermodynamics for such processes to take place both at a finite rate and nevertheless re-

1 Tolman, Proc. Nat. Acad. Sci. 14, 268 (1928); ibid. 14, 701 (1928); Phys. Rev. 35, 875 (1930); ibid. 35, 896 (1930).

${ }^{2}$ See references 3 to 8 .

3 Tolman, Phys. Rev. 35, 904 (1930); Tolman and Ehrenfest, Phys. Rev. 36, 1791 (1930).

${ }^{4}$ Tolman, Proc. Nat. Acad. Sci. 14, 348, 353 (1928); ibid. 17, 153 (1931). 
versibly without increase in entropy, owing to an opportunity for correlated changes in entropy density and gravitational field not contemplated in the older thermodynamics. This possibility for reversible changes at a finite rate was illustrated by studies of three different systems, - a non-static universe filled solely with black-body radiation, ${ }^{5}$ - a non-static universe filled with incoherent matter exerting no pressure, ${ }^{6}$ - and a non-static universe filled with a mixture of monatomic gas in equilibrium with black-body radiation. ${ }^{7}$ In all three cases it was shown that the systems could expand or contract at a finite rate and nevertheless reversibly without increase in entropy, the latter of the three, however, only under the special assumption that the gas remain in equilibrium with the radiation. It was also shown that the entirely reversible expansion of such universes would be accompanied by a number of phenomena, such as the outward flow of radiation, which are similar to processes that have ordinarily been interpreted as irreversible. Finally, the possibility for the periodic expansion and contraction of these systems was investigated, since any thermodynamic obstacles to such behaviour are evidently eliminated by the demonstrated reversibility. ${ }^{8}$ It was found that no strictly periodic solutions for the equations of motion of such systems could exist, but that nevertheless quasi-periodic solutions would be possible such that the system would expand from zero proper volume to a maximum and return once more to the starting point without increase in entropy, the solution failing however at this exceptional point. It was emphasized, moreover, in view of the physics of the situation, that the return of such a system to zero volume without increase in entropy could be followed by a duplicate repetition of the expansion even though the over-idealized analysis fails to carry through the exceptional point of zero volume.

In contrast to these previous applications to systems which are in static equilibrium, and to non-static systems in which reversible processes are taking place, it is the purpose of the present article to apply the principles of relativistic thermodynamics to non-static systems in which irreversible processes take place. Such an extension in the range of application may have considerable interest for the interpretation of cosmological phenomena, since, even though it has been shown that reversible changes can take place at a finite rate in such a way as to give some phenomena which are similar to important ones in the actual universe, it is evident that reversible changes form only a limited class of the totality of conceivable thermodynamic processes.

The special task of this article will be to examine an essential change in point of view which becomes necessary when the effects of irreversible processes in isolated systems are to be studied by the new methods of relativistic thermodynamics. The necessity for the change arises primarily because of the well-known conclusion that the principle of the conservation of energy cannot hold in the mechanics of general relativity in the same simple form as in

5 Tolman, Phys. Rev. 37, 1639 (1931).

${ }^{6}$ This was discussed in connection with the work on periodicity; see reference 8 .

7 Tolman, Phys. Rev. 38, 797 (1931).

8 Tolman, Phys. Rev. 38, 1758 (1931). 
the classical mechanics. On account of this difference, we shall find in relativistic thermodynamics, that the possible increases in entropy which accompany the occurrence of irreversible processes in an isolated system are not subject to a limitation such as is imposed in the classical thermodynamics by a fixed constant value for the energy of the system. Indeed we shall find in relativistic thermodynamics a possibility for irreversible processes to take place in an isolated system without ever reaching a condition of maximum entropy and minimum free energy where further change would be impossible.

We may now proceed to the detailed examination of our problem. In the next section, $\$ 2$, we shall first obtain some expressions, giving the dependence of entropy on the variables which determine the state of a system, in a form which will be suitable for our further work. These expressions will be derived by the methods of classical thermodynamics, but, in accordance with the principle of equivalence, will also be valid in relativistic thermodynamics when applied to an infinitesimal region using proper coordinates. With the help of these expressions, we can then explain in detail in $\$ \$ 3,4$ the reason why the failure of the principle of energy conservation in its old simple form does provide hitherto unsuspected possibilities for a continuous increase in the entropy of an isolated system without ever reaching an unsurpassable maximum. In $\$ 5$ we shall then show that such possibilities could actually be realized in the case of a considerable class of non-static models of the universe. And in $\$ 6$ we shall consider in more detail a particular one of these models which contains a mixture of monatomic gas and black-body radiation which do not remain in equilibrium, in other words a non-static model of the universe with possibilities for irreversible annihilation of matter instead of the reversible annihilation previously discussed. Finally in $\$ 7$ we shall make a few remarks concerning the possible bearing of our findings on the phenomena of the actual universe.

\section{§2. Dependence of Entropy on the State of a System}

In the present section we shall obtain some expressions for the dependence of entropy on the state of a system which will be needed for our further work. The expressions will be obtained by the methods of the classical thermodynamics, which tacitly assumes flat space-time, and will be found to be valid in the curved space-time of general relativity only when applied to an infinitesimal region using proper coordinates.

We shall limit our consideration to simple systems, having uniform temperature, pressure and composition throughout, so that the state of the system can be completely specified by a statement of the energy $E$, volume $v$, and number of molecules $N_{1} \cdots N_{n}$, of the different substances which the system contains. Since the entropy $S$ of the system is a function of its state we can then evidently write in accordance with the principles of the differential calculus

$$
d S=\frac{\partial S}{\partial E} d E+\frac{\partial S}{\partial v} d v+\frac{\partial S}{\partial N_{1}} d N_{1}+\cdots+\frac{\partial S}{\partial N_{n}} d N_{n} .
$$


For an infinitesimal change in energy and volume, however, keeping the composition constant, we have for the change in entropy the familiar expression

$$
d S=\frac{1}{T} d E+\frac{p}{T} d v
$$

which gives us for the partial differenials of entropy with respect to energy and volume the well-known values

$$
\frac{\partial S}{\partial E}=\frac{1}{T} \text { and } \quad \frac{\partial S}{\partial v}=\frac{p}{T}
$$

where $T$ and $p$ are temperature and pressure, and substituting above can write our first equation in the more useful form ${ }^{9}$

$$
d S=\frac{1}{T} d E+\frac{p}{T} d v+\frac{\partial S}{\partial N_{1}} d N_{1}+\cdots+\frac{\partial S}{\partial N_{n}} d N_{n}
$$

Two other forms of this equation will also be useful. As an expression for the entropy of the system in terms of the Helmholtz free energy $A$ we have by the equation of definition

$$
S=\frac{E-A}{T}
$$

and in terms of the thermodynamic potential, or free energy $F$ as used by chemists, we have

$$
S=\frac{E+p v-F}{T}
$$

and by differentiation can write

and

$$
d S=\frac{1}{T} d E-\frac{1}{T} d A-\frac{S}{T} d T
$$

$$
d S=\frac{1}{T} d E+\frac{p}{T} d v+\frac{v}{T} d p-\frac{1}{T} d F-\frac{S}{T} d T
$$

Equating the expression for $d S$ given by (4) with those given by (6a) and (6b), and solving for $d A$ and $d F$, we then easily obtain

and

$$
d A=-S d T-p d v-T \frac{\partial S}{\partial N_{1}} d N_{1}-\cdots-T \frac{\partial S}{\partial N_{n}} d N_{n}
$$

$$
d F=-S d T+v d p-T \frac{\partial S}{\partial N_{1}} d N_{1}-\cdots-T \frac{\partial S}{\partial N_{n}} d N_{n}
$$

${ }^{9}$ Allowing for the difference in notation this equation is entirely equivalent to a fundamental equation upon which Gibbs based his work on the equilibrium of heterogeneous substances. See his "Collected Works" Longmans, Green and Company (1928) Vol. I, Eq. (12), page 63. 
Examining these equations, it is evident that if we regard the Helmholtz free energy $A$ as a function of temperature, volume and composition, and the thermodynamic potential $F$ as a function of temperature, pressure and composition we can write

$$
\left(\frac{\partial A}{\partial N_{i}}\right)_{T, v}=\left(\frac{\partial F}{\partial N_{i}}\right)_{1, p}=-T\left(\frac{\partial S}{\partial N_{i}}\right)_{E, v} .
$$

Where the subscripts indicate the independent variables, in addition to those giving the composition $N_{1} \cdots N_{n}$, which are regarded as determining the quantities $A, F$, and $S$.

With the help of (8) we can now re-express our fundamental Eq. (4) in two further useful forms, giving us altogether the three expressions

$$
\begin{aligned}
d S & =\frac{1}{T} d E+\frac{p}{T} d v+\left(\frac{\partial S}{\partial N_{1}}\right)_{E, v} d N_{1}+\cdots+\left(\frac{\partial S}{\partial N_{n}}\right)_{E, v} d N_{n} \\
d S & =\frac{1}{T} d E+\frac{p}{T} d v-\frac{1}{T}\left(\frac{\partial A}{\partial N_{1}}\right)_{T, v} d N_{1}-\cdots-\frac{1}{T}\left(\frac{\partial A}{\partial N_{n}}\right)_{T, v} d N_{n} \\
d S & =\frac{1}{T} d E+\frac{p}{T} d v-\frac{1}{T}\left(\frac{\partial F}{\partial N_{1}}\right)_{T, p} d N_{1}-\cdots-\frac{1}{T}\left(\frac{\partial F}{\partial N_{n}}\right)_{T, p} d N_{n}
\end{aligned}
$$

\section{§3. Limitation on Entropy Increase Imposed in Classical Thermodynamics by Conservation of Energy}

With the help of these equations we can now see clearly the reasons why the classical thermodynamics has accustomed us to expect that the occurrence of irreversible processes in an isolated system would ultimately lead to a condition of maximum entropy and minimum free energy such that no further change would be possible.

For example, we could consider our isolated system either to be a coherent mass of solid or liquid situated somewhere in free space, or to be a mass of gas situated in free space and prevented from escaping by enclosure in an idealized container, having constant volume but otherwise adding nothing to the thermodynamic properties of the system of actual interest. We could then apply Eq. (9a) to analyze the increase in entropy which must accompany the occurrence of irreversible processes in such systems.

Considering the variables $E, v, N_{1} \cdots N_{n}$, to be changed one at a time from their initial to their final values in order to calculate the increase in entropy $\Delta S$ which accompanies the change in state of the system, we should conclude that the first term on the right hand side of Eq. (9a) could contribute nothing to the increase in entropy, since we should have

$$
\int \frac{d E}{T}=0
$$

owing to the constant value of $E$ for an isolated system prescribed by the classical principle of the conservation of energy; and we should also conclude that the second term could contribute nothing, since we should have 


$$
\int \frac{p d v}{T}=0
$$

owing to the value of the pressure $p=0$ in the case of the coherent mass, and owing to the constant volume of the idealized container in the case of the gas. Hence we should be forced to the conclusion that the actual increase in entropy accompanying irreversible processes in our system could be calculated solely from the last terms in Eq. (9a) in accordance with the expression

$$
\Delta S=\int\left[\left(\frac{\partial S}{\partial N_{1}}\right)_{E, v} d N_{1}+\cdots+\left(\frac{\partial S}{\partial N_{n}}\right)_{E, v} d N_{n}\right] .
$$

The possibilities for increase in entropy, however, provided by this last equation are limited, since for our isolated systems of constant energy it is evident that there is only a restricted range of possible adjustment for the variables $N_{1}$ to $N_{n}$ which determine the composition. Hence when the most favorable adjustment has been reached the entropy will be at a maximum and further change cannot take place. In addition it is to be noted by comparing the equivalent forms (9a), (9b) and (9c), that in this final state of the system not only will the entropy be a maximum, but the Helmholtz free energy $A$ will be a minimum for adjustments in composition at constant temperature and volume, and the thermodynamic potential or chemists' free energy $F$ will be a minimum for adjustments in composition at constant temperature and pressure. It is by considerations such as these that the classical thermodynamics has led to the belief that the ultimate state of an isolated system would be a condition of maximum entropy and minimum free energy, where possibilities for further change or for the performance of useful work would no longer exist.

\section{\$4. Possibility Provided in Relativistic Thermodynamics for Removal} of Limitation on Entropy Increase Imposed by the

\section{Conservation of Energy}

We may now turn to an analysis of this same problem using the methods of relativistic thermodynamics. To carry this out, we cannot forthwith apply Eqs. (9) to our isolated system as a whole to calculate the entropy increases which accompany the irreversible processes that take place in it, since our reason for resorting at all to a relativistic treatment lies in the consideration that gravitational action in producing a deviation from the flat space-time of the classical theory may have an important effect on our results; and in the curved space-time of general relativity such quantities as the entropy, energy, and volume of a system need special interpretation.

In accordance with the principles of relativistic thermodynamics, however, which I have previously developed, ${ }^{10}$. we can define the entropy of a finite system by the expression

\footnotetext{
${ }^{10}$ See reference 1 , last article $\$ 3$.
} 


$$
S=\iiint \phi_{0} \sqrt{-g} \frac{d x_{4}}{d s} d x_{1} d x_{2} d x_{3}
$$

and by applying the second law of thermodynamics in its relativistic form obtain as a necessary condition for thermodynamic change in an isolated system

$$
\frac{\partial S}{\partial x_{4}}=\iiint \frac{\partial}{\partial x_{4}}\left(\phi_{0} \sqrt{-g} \frac{d x_{4}}{d s}\right) d x_{1} d x_{2} d x_{3} \geqq 0
$$

where $x_{1}, x_{2}$ and $x_{3}$ are the spatial coordinates, $x_{4}$ is the time coordinate, $\phi_{0}$ is the proper density of entropy as measured by a local observer, $g$ is the determiriant formed from the gravitational potentials, $d x_{4} / d s$ is the ratio of increase in coordinate time to increase in proper time at the point in question, and the integration is to be taken over the whole range of spatial coordinates necessary to include the system.

The two signs of inequality and equality in the above expression refer respectively to the case of irreversible and reversible processes, and we may now limit ourselves to the former since our present interest lies in irreversible processes. Furthermore, for our present purposes it will be sufficient to consider cases where the general inequality given by (14) is satisfied by having the inequality

$$
\frac{\partial}{\partial x_{4}}\left(\phi_{0} \sqrt{-g} \frac{d x_{4}}{d s}\right)>0
$$

itself hold at each point in our thermodynamic fluid. ${ }^{11}$

The physical interpretation of this expression can be made more evident, however, if we choose our coordinate system so that the thermodynamic fluid under consideration is everywhere at rest in the spatial coordinates $x_{1}$, $x_{2}$ and $x_{3}$. Since the macroscopic velocities $d x_{1} / d s, d x_{2} / d s$ and $d x_{3} / d s$ will then everywhere be zero, it is evident that the amount of fluid in any given coordinate range $\delta x_{1} \delta x_{2} \delta x_{3}$ will not be changing with the time; and in accordance with the principles of relativity we can write for the proper volume $\delta v_{0}$ of the small element of fluid in such a range the well known equation

$$
\delta v_{0}=\sqrt{-g} \frac{d x_{4}}{d s} \delta x_{1} \delta x_{2} \delta x_{3}
$$

and, by substituting above, rewrite expression (15) in the form

$$
\frac{d}{d x_{4}}\left(\phi_{0} \sqrt{-g} \frac{d x_{4}}{d s} \delta x_{1} \delta x_{2} \delta x_{3}\right)=\frac{d}{d x_{4}}\left(\phi_{0} \delta v_{0}\right)=\frac{d S_{0}}{d x_{4}}>0
$$

11 This has the effect of limiting our present considerations to cases where the proper entropy of each element of the fluid will be found increasing with the time when measured by a local observer, without reference to the flow of heat in or out of that element. (See expression 17). If flow of heat were taking place out of an element, we could have irreversibility even without an increase in the proper entropy of the element, but this will not be of interest for our present purposes since the later work of this paper will deal only with systems where there is no flow of heat. In any case there is of course no loss in rigour in using (15) since we are only interested now in having a sufficient condition for irreversibility. 
We thus obtain as a sufficient condition for the occurrence of irreversible processes in our isolated system the very satisfactory result that the proper entropy, $S_{0}=\phi_{0} \delta v_{0}$, of each element of fluid as measured by a local observer shall increase with the time. ${ }^{12}$

This simple condition, moreover, is now in a very satisfactory form for purposes of calculation since the proper entropy of a small element of the fluid will depend on its proper energy, $E_{0}=\rho_{00} \delta v_{0}$, proper pressure $p_{0}$, proper temperature $T_{0}$, and number of molecules in the element $N_{1} \cdots N_{n}$, in the same way as in the classical thermodynamics. Hence, by analogy with Eq. (4), we can now write

$$
d S_{0}=\frac{1}{T_{0}} d E_{0}+\frac{p_{0}}{T_{0}} d\left(\delta v_{0}\right)+\frac{\partial S_{0}}{\partial N_{1}} d N_{1}+\cdots+\frac{\partial S_{0}}{\partial N_{n}} d N_{n}
$$

as an expression which gives the dependence of the proper entropy of our small element of fluid on the variables which determine its state. And our sufficient condition for irreversible processes will be met if the proper entropy of each small element of the fluid, as calculated with the help of Eq. (18), increases with the time.

At first sight it might appear as though Eq. (18) provided no more opportunity than our former classical Eq. (4) for a continuous increase in entropy without reaching an unsurpassible maximum corresponding to the most favorable values for the variables $N_{1} \cdots N_{n}$. And from the classical point of view this would seem to be the case, since attempts to adjust the other variables $E_{0}$ and $\delta v_{0}$ so as to obtain a greater increase in entropy would appear to be of no avail, as the energy and volume of one element of the fluid would have to be increased at the expense of other elements.

In relativistic mechanics, however, it is well known that the principle of the conservation of energy does not hold in the simple form which would require a constant value for the total proper energy of an isolated system. The quantity analogous to energy which is actually conserved in relativistic mechanics is ${ }^{13}$

$$
\iiint\left(\mathfrak{T}_{4}^{4}+\mathbf{t}_{4}^{4}\right) d x_{1} d x_{2} d x_{3}=\text { const. }
$$

where $\mathfrak{I}_{4}^{4}$ is the energy-momentum tensor-density and $\mathfrak{t}_{4}^{4}$ is the pseudo tensor-density of potential energy and momentum. In proper coordinates the first term of this expression reduces to proper energy

$$
\mathfrak{T}_{4}^{4} d x_{1} d x_{2} d x_{3}=\rho_{00} \delta v_{0}=E_{0}
$$

and the second term reduces to zero, but in general proper coordinates cannot be used throughout the whole system, and in general the total proper energy of an isolated system is not a constant in relativistic mechanics.

${ }^{12}$ Since an increment in proper time $d t_{0}$ and the corresponding increment in coordinate time $d x_{4}$ are both to be taken as having the same sign, the inequality (17) is also true for a local observer.

${ }^{13}$ See for example, Tolman, the third article in reference 1. 
Hence in relativistic thermodynamics we must investigate the possibility that a continuous increase in the proper entropy of each element of an isolated system could be brought about by increases in the proper energy of the elements, without every reaching an unsurpassable maximum. In the next section it will be shown by an illustrative example, that this new possibility for the continuous occurrence of irreversible processes without reaching a final state of quiescence, might actually be realized in a way which is not trivial.

\section{\$5. Possibility for Continuous Increase in Entropy in a Non- Static Universe if the Cosmological Constant is Zero or Negative}

\section{a. The mechanics of the model.}

As an isolated system to illustrate the possibility for the continuous occurrence of irreversible processes, we shall choose a non-static model of the universe filled with a homogeneous distribution of matter and energy. ${ }^{14}$ The line element for such a non-static universe can be derived ${ }^{15}$ by treating the contents of the model for purposes of large scale considerations as a perfect fluid, and written in the form

$$
d s^{2}=-\frac{e^{g(t)}}{\left[1+r^{2} / 4 R^{2}\right]^{2}}\left(d r^{2}+r^{2} d \theta^{2}+r^{2} \sin ^{2} \theta d \phi^{2}\right)+d t^{2}
$$

where $r, \theta$ and $\phi$ are the spatial coordinates, $t$ is the time coordinate, $R$ is a constant, and the dependence of the line element on the time is given by the exponent $g(t)$.

With this choice of coordinates, particles at rest in the coordinate system will not be subject to acceleration but will remain permanently without spatial velocity. Hence the choice of coordinate system is such that we may regard the thermodynamic fluid filling the model as macroscopically at rest with respect to the spatial coordinates, and can apply expressions (17) and (18) of the preceding section to the entropy of any small element of this fluid. For the proper volume of a small element of the fluid contained in the coordinate range $\delta r \delta \theta \delta \phi$, we may evidently write

$$
\delta v_{0}=\frac{e^{3 \theta / 2}}{\left[1+r^{2} / 4 R^{2}\right]^{3}} r^{2} \sin \theta \delta r \delta \theta \delta \phi
$$

and this in general will be changing with the time, if $g(t)$ is not a constant. In accordance with the above, however, the contents of the element will not be affected by any net flow of particles across its boundaries.

Of the possible non-static models which could agree with the line element (21), the special class which will illustrate our considerations will be obtained if the constant $R$ occurring in the line element is real, and the cosmological constant $\Lambda$ occurring in Einstein's relation between the energy-momentum

${ }^{14}$ For a partial historical account of various treatments which have been given to the non-static line element for the universe, see Tolman, Proc. Nat. Acad. 16, 582 (1930).

${ }^{15}$ See Tolman, Proc. Nat. Acad. Sci. 16, 320 (1930). 
tensor and the curvature of space-time is not a positive quantity. If $R$ is real the "radius" of the model $R e^{g / 2}$ will be real and the universe closed. As to the cosmological constant $\Lambda$, our only experimental evidence is that it must be small enough not to affect phenomena in the solar system. If we take it zero the equations of relativistic mechanics are somewhat simplified, but there is one point in our later development where a small negative value might seem preferable. These restrictions, $R$ real and $\Lambda=0$ or $\Lambda<0$, will be adopted in what follows.

We are now ready to consider the behaviour of this class of non-static models. Taking the material filling the model as a perfect fluid of proper macroscopic density $\rho_{00}$ and proper pressure $p_{0}$, the components of the energy-momentum tensor corresponding to the line element (21) have been worked out, ${ }^{16}$ and with $\Lambda=0$ give for the pressure and density the values

$$
\begin{aligned}
& 8 \pi p_{0}=\left(-8 \pi T_{i}^{i}\right)=-\frac{1}{R^{2}} e^{-g}-\ddot{g}-\frac{3}{4} \dot{g}^{2} \quad i=(1,2,3) \\
& 8 \pi \rho_{00}=\left(8 \pi T_{4}^{4}\right)=\frac{3}{R^{2}} e^{-g}+\frac{3}{4} \dot{g}^{2}
\end{aligned}
$$

together with

$$
T_{\mu}^{\nu}=0 \quad(\mu \neq \nu)
$$

where the dependence of the pressure and density on the time is given through their dependence on the exponent $g(t)$ and its first and second time derivatives $\dot{g}$ and $\ddot{g}$. Furthermore, substituting the expressions for $T_{\mu}^{\nu}$ in the fundamental equation of relativistic mechanics, which for this purpose can be expressed most conveniently in the form

$$
\frac{\partial \mathfrak{T}_{\mu}^{\nu}}{\partial x_{\nu}}-\frac{1}{2} \mathfrak{T}^{\alpha \beta} \frac{\partial g_{\alpha \beta}}{\partial x_{\mu}}=0
$$

we obtain ${ }^{17}$ for the case $\mu=4$

$$
\frac{d}{d t}\left[\frac{\rho_{00} e^{3 g / 2}}{\left(1+r^{2} / 4 R^{2}\right)^{3}}\right]+p_{0} \frac{d}{d t}\left[\frac{e^{3 g / 2}}{\left(1+r^{2} / 4 R^{2}\right)^{3}}\right]=0
$$

which, since $r$ and $R$ are independent of $t$, can also be written in the simple form,

$$
\frac{d}{d t}\left(\rho_{00} e^{3 g / 2}\right)+p_{0} \frac{d}{d t}\left(e^{3 g / 2}\right)=0
$$

and in addition by noting the expression for proper volume given by (22), can be written in the specially illuminating form

$$
\frac{d}{d t}\left(\rho_{00} \delta v_{0}\right)+p_{0} \frac{d}{d t}\left(\delta v_{0}\right)=0
$$

${ }^{16}$ See reference 15, Eqs. (34).

${ }^{17}$ See Tolman, Proc. Nat. Acad. Sci. 16, 409 (1930), Eq. (4). 
This last form shows that, as the exponent $g$ changes with the time, the behaviour of each element of the fluid is such that its increase in proper energy can be regarded as the work done on it by its surroundings. In other words as time proceeds each element of the fluid may be regarded as subjected to an adiabatic ${ }^{18}$ expansion or compression, according as $g$ is increasing or decreasing with the time.

We can also obtain information as to the behaviour of the model as a whole over long periods of time. Solving for $\ddot{g}$ the equation for pressure given by (23), we obtain

$$
\ddot{g}=-\frac{1}{R^{2}} e^{-g}-\frac{3}{4} \dot{g}^{2}-8 \pi p_{0} .
$$

Since $R$ is real by hypothesis and the pressure $p_{0}$ could not be negative for the kinds of fluid with which we shall wish to regard the universe as filled, we see that the acceleration $\ddot{g}$ would always be negative, and hence, if the model were expanding, that $g$ and the "radius" $R e^{g / 2}$ would ultimately reach maximum values and the model would start contracting. The only possible exception to such behaviour would perhaps be a special case in which $g$ would become infinite and $g$ and $p_{0}$ zero at the upper limit. ${ }^{19}$ Even this possibility, however, could be ruled out, by ascribing a small negative value to the cosmological constant $\Lambda$, since the equation corresponding to (28) when $\Lambda$ is not omitted reads ${ }^{20}$

$$
\ddot{g}=-\frac{1}{R^{2}} e^{-g}-\frac{3}{4} \ddot{g}^{2}-8 \pi p_{0}+\Lambda
$$

and with $\Lambda$ a negative constant, the acceleration would always be negative even if $g$ went to infinity and $g$ and $p_{0}$ to zero.

After the model has passed its point of maximum expansion, then in accordance with (28) the negative acceleration would get greater and greater and the contraction would continue at an increasing rate to zero proper volume with $g=-\infty$ and the "radius" $R e^{g / 2}=0$. The equations of motion fail to carry us through this exceptional point, perhaps because of an over idealization of the model. ${ }^{21}$ From a physical point of view it is evident, however, that this contraction to the lower limit could only be followed by renewed expansion. ${ }^{22}$

We thus see that relativistic mechanics appears to provide within its framework conceivable models of non-static universes which would undergo a continued succession of expansions and contractions. In any case we shall assume this possibility in what follows.

${ }^{18}$ Note that in English usage the word "adiabatic" applies to a change in state with no flow of heat into the system, without reference to whether the change is a reversible or irreversible one.

${ }^{19}$ In a later paper my colleague Professor Morgan Ward and I hope to show that this exceptional case would not occur even with the cosmological constant $\Lambda$ exactly equal to zero.

${ }^{20}$ See reference 15, Eqs. (34).

${ }^{21}$ Compare, Einstein, Berl. Ber., 1931, p. 235.

${ }^{22}$ Compare, Tolman, reference 8 . 


\section{b. The thermodynamics of the model.}

We must now inquire into the thermodynamics of such models, since from the classical point of view we have become accustomed to the idea that there must be some thermodynamic hindrance to such a continued succession of expansions and contractions in an isolated system. From the relativistic point of view, however, it appears that no such hindrance could exist, since we were led to the conclusion that the series of expansions and contractions would take place solely from the fact that the expressions (28)-(28a) prescribe a negative value for the acceleration $\ddot{g}$ so that expansion will always be followed by contraction. And, if we fill our model by hypothesis with a material that cannot exert a negative pressure, ${ }^{23}$ it is evident that this negative value of $\ddot{g}$ would obtain without reference to the thermodynamic nature of the reversible or irreversible processes taking place inside the model.

It hence appears in general that relativistic thermodynamics could not impose restrictions which would prevent such a series of expansions and contractions. It will also be of interest, however, to analyze the thermodynamics of our model in somewhat more detail.

In the first place, it is of course immediately evident that there would be no restriction imposed on the succession of expansions and contractions provided they took place reversibly without increase in entropy, since the model would then arrive at the lower limit of contraction with unchanged entropy, and a renewed expansion identical with the previous one could be initiated. Such behaviour, which becomes of interest since relativistic thermodynamics provides new possibilities for reversible changes at a finite rate, ${ }^{24}$ has already been discussed in a previous article..$^{25}$

In the present article, however, we are primarily interested in any thermodynamic hindrance to the succession of expansions and contractions which might arise because of irreversible processes. To analyze this point we may best consider the behaviour of a small element of the fluid which we take as filling our model. In accordance with Eq. (27) as $g(t)$ increases and decreases with the time, such an element would be subjected to a series of adiabatic expansions and compressions without flow of heat from the outside and with perfect balance between internal and external pressure, because of the uniformity of $p_{0}$ throughout the model. Irreversibility with its accompanying increase in entropy could arise, however, on account of internal changes within the element, and in general this would presumably occur since the expansions and contractions would take place at a finite rate, and the internal variables, in our case the quantities $N_{1} \cdots N_{n}$ which determine the composition of the fluid, would adjust themselves to the changing volume with a certain lag behind the condition of equilibrium, thus leading to an increase in the entropy of the element.

It is evident, however, that such behaviour would provide nothing which

${ }^{23}$ Note that the non-negative pressure would be necessary only in the last stages of expansion.

${ }^{24}$ Reference 5.

25 Reference 8. 
would make the continued expansion and contraction impossible. Except for the absence of friction and the balance between internal and external pressure, the behaviour of the fluid in the element is similar to the classical behaviour of a fluid placed in a cylinder with non-conducting walls and subjected to a series of compressions and expansions with the help of a movable piston. Classically the entropy of the fluid would increase because of the lag in the adjustment of the internal variables determining the state of the system behind their equilibrium values. And this increase in entropy would continue as long as the expansions and contractions were continued, without of itself preventing us from subjecting the fluid to further expansions and contractions, provided we have the desire to do so and the mechanical energy which may be needed to move the piston. Similarly in the relativistic case, if the equations of relativistic mechanics do inevitably prescribe a continued succession of expansions and contractions for a particular class of non-static models of the universe, it is evident that the occurrence of irreversible processes inside the model furnishes no thermodynamic preventive to such behaviour.

Before turning from this general discussion of the thermodynamic behaviour of the class of models under consideration to the discussion of any special model, it will also be of interest to analyze somewhat more in detail the mechanism by which entropy increases occur in the elements of our fluid, and show a general type of relation which exists between the entropy content and energy content of an element. In accordance with Eq. (18) we can write for the rate with which the entropy of any given element is increasing with the time

$$
\frac{d S_{0}}{d t}=\frac{1}{T_{0}} \frac{d E_{0}}{d t}+\frac{p_{0}}{T_{0}} \frac{d\left(\delta v_{0}\right)}{d t}+\left(\frac{\partial S_{0}}{\partial N_{1}}\right) \frac{d N_{1}}{d t}+\cdots+\left(\frac{\partial S_{0}}{\partial N_{n}}\right) \frac{d N_{n}}{d t} .
$$

It is evident from this that the immediate occasion for the entropy increase taking place at any given time in an element must lie in the changes taking place in the variables $N_{1} \cdots N_{n}$, which determine the composition, since for the first two terms above we can write

$$
\frac{1}{T_{0}} \frac{d E_{0}}{d t}+\frac{p_{0}}{T_{0}} \frac{d\left(\delta v_{0}\right)}{d t}=0
$$

owing to the adiabatic character of the change given by Eq. (27). The changes in the variables $N_{1} \cdots N_{n}$, however, can contribute to a positive rate of entropy increase since they will occur in the direction towards equilibrium under conditions where the partial differentials $\left(\left(\partial S_{0} / \partial N_{1} \cdots \partial S_{0} / \partial N_{n}\right)\right.$ do not have their equilibrium values. For example if the fluid were a mixture of diatomic gas and the elements, into which it dissociates in accordance with the reaction

$$
A_{2}=2 A
$$

and the rate of expansion were too great for the dissociation to keep pace, we should have $A_{2}$ breaking up into $2 A$ under non-equilibrium conditions and 
this would lead to an increase in entropy. We thus see that the immediate mechanism by which the entropy increase actually takes place is through the adjustment of the internal variables $N_{1} \cdots N_{n}$ which determine the composition.

On account of this fact that the actual mechanism of entropy increase lies in the adjustment of composition, it might at first sight be supposed that the continuous increase in entropy would require no change in the energy of the element. In the long run, however, this is not the case. Let us consider the condition of an element of the fluid at two different instants $t_{1}$ and $t_{2}$ which are a long time apart so that there has been a very great increase in its entropy, and for simplicity let us pick $t_{2}$ such that $g(t)$ for the universe and $\delta v_{0}$ for the element have their earlier values. We could then use Eq. (18)

$$
d S_{0}=\frac{1}{T_{0}} d E_{0}+\frac{p_{0}}{T_{0}} d\left(\delta v_{0}\right)+\frac{\partial S_{0}}{\partial N_{1}} d N_{1}+\cdots+\frac{\partial S_{0}}{\partial N_{n}} d N_{n}
$$

to calculate the difference in entropy of the two states, by considering the effect of changing the variables one at a time from their initial to their final values. If the increase in entropy has been large it is then evident that the proper energy $E_{0}$ will have to be greater in the final state than in the initial state, since $\delta v_{0}$ is taken the same in the two states, and the adjustment of the composition at constant $E_{0}$ and $\delta v_{0}$ could only lead, as already discussed in $\S 3$, to a limited increase in entropy.

We hence conclude that in the long run we must expect the proper energy of each element of the fluid filling the model to increase with the time. The mechanism by which this occurs will of course have to lie in a general tendency for the pressure $p_{0}$ to be on the average greater during a compression than it was during the preceding expansion. This, however, is in qualitative accord with the behaviour that we should expect if the composition lags in adjusting itself to equilibrium. We thus see that although the actual mechanism of entropy increase lies in the adjustment of composition, which sometimes takes place in one direction and sometimes in the other according as conditions are changed, nevertheless in the long run the continued increase in entropy involves and is made possible by an increase in proper energy.

The possibility for such an increase in proper energy is, as discussed in $\S 4$, a characteristic feature of relativistic as distinguished from classical mechanics. For example let us return once more to our comparison between the classical behaviour of a fluid placed in a cylinder with non-conducting walls and subjected to a series of expansions and contractions, and the relativistic behaviour of an element of the fluid in our expanding and contracting universe. From the classical point of view, it would not be the increase in the entropy of the fluid in the cylinder per se, which would prevent us from subjecting it to further expansions and compressions, but the fact that ultimately we should expect to have no further external mechanical energy available for completing another compression. In the relativistic case, however, the laws of mechanics do not require any constancy in the total supply of proper energy. Indeed in accordance with Eq. (27), 


$$
\frac{d}{d t}\left(\rho_{00} \delta v_{0}\right)+p_{0} \frac{d}{d t}\left(\delta v_{0}\right)=0
$$

if $p_{0}$ is positive, the proper energies of all elements of the fluid are decreasing when the model is expanding and increasing when it is contracting, and hence in the long run there can be a tendency for the total proper energy to increase, if the pressure on compression tends to be higher than on expansion.

Noting the expressions for proper volume and proper density of energy given by (22) and (23), it is evident that the proper energy of each element of the fluid and the total proper energy of the model will be proportional to

$$
8 \pi \rho_{00} e^{3 g / 2}=\frac{3}{R^{2}} e^{g / 2}+\frac{3}{4} e^{3 g / 2} \dot{g}^{2} .
$$

Hence in a long series of expansions and contractions we must expect $g(t)$ and the "radius" of the model $R e^{g / 2}$ to become greater and greater at succeeding times when the velocity $g$ has the same values, for example at succeeding points of maximum expansion. Similarly for succeeding points where $g(t)$ and the "radius" $R e^{g / 2}$ have their earlier values we must expect the velocities $\dot{g}$ to get greater. This permits a rough plot of the "radius" $R e^{g / 2}$ against the time for any model of the class under consideration - the upper limit of the "radius" to which the model expands gradually getting greater and greater, and its rate of change at a given value also getting greater.

\section{§6. Model of Non-Static Universe with Irreversible Annihilation of Matter}

In a previous paper ${ }^{26}$ I have treated a non-static model of the universe, containing a mixture of perfect monatomic gas and black-body radiation, which were taken to be capable of conversion one in to the other. Making the hypothesis that equilibrium was maintained between the matter and radiation, it was shown that such a model could expand or contract reversibly at a finite rate, with a reversible annihilation of matter (i.e., conversion into radiation) during expansion and a reversible formation of matter during compression. With the help of our present considerations, we may now treat such a non-static model, on the perhaps more probable hypothesis that the interconversion does not have a high enough rate to maintain equilibrium-in other words, a non-static model of the universe with the possibility for irreversible annihilation of matter.

In such a model if we start expanding from a point at which the matter and radiation are at their equilibrium concentrations, or better pass through such a point in the course of an expansion, then as the expansion continues matter will tend to go over into radiation, ${ }^{27}$ and, if this cannot take place rapidly enough to maintain equilibrium concentrations, irreversibility will re-

${ }^{26}$ Reference 7.

${ }^{27}$ See Eqs. (36) and (38), reference 7. We pick for our illustration a case where the temperature does not have the extremely high values where formation of matter accompanies expansion. 
sult and the changes of matter into radiation that do occur will be accompanied by an increase in entropy. In accordance with our general treatment of the mechanics of this class of models, the expansion will continue to a maximum point where contraction will set in. As the contraction proceeds, the matter will still be for a time in excess of its equilibrium concentration and will continue to change into radiation, thus making the pressure higher on the return path than at the same volume during the expansion. As the contraction proceeds still further, however, a point will be reached where we again pass through new equilibrium values for the concentrations, immediately followed by conditions such that radiation will now be going over into matter irreversibly with increase in entropy, the actual pressure of the radiation being now higher than would correspond to equilibrium. We thus see in a general way the mechanism by which increase in entropy and increase in proper energy would take place in such a model.

We may also draw certain conclusions as to the long range behaviour of the model. For the proper entropy $S_{0}$ of an element $\delta v_{0}$ of the fluid filling the model, we may evidently write the sum of the entropies of the gas and radiation in the element

$$
S_{0}=\frac{3}{2} N k \log T_{0}+N k \log \frac{\delta v_{0}}{N}+N k \log b e^{5 / 2}+\frac{4}{3} a T_{0}{ }^{3} \delta v_{0}
$$

where $N$ is the number of atoms in the element, $k$ is Boltzmann's constant, $a$ is Stefan's constant, and $b$ is a constant of the right magnitude to assure the same starting point for the entropy of the gas and the radiation when their interconversion is to be considered, the form of the term containing $b$ being chosen in such a way as to give a simple form to the expression for the equilibrium concentration of gas, which can be shown to be ${ }^{28}$

$$
\frac{N}{\delta v_{0}}=b T_{0}^{3 / 2} e^{-m c 2 / k T_{0}}
$$

where $m$ is the mass per atom and $c$ is the velocity of light.

By using the ordinary methods for determining a maximum, it can easily be shown from Eq. (35) that at a given temperature $T_{0}$ and a given volume $\delta v_{0}$, the proper entropy $S$ would have its maximum possible value with the composition

$$
N=b e^{3 / 2} T^{3 / 2} \delta v_{0}
$$

And substituting this value into (35) we obtain in general for the proper entropy of an element

$$
S_{0} \leqq\left(b k e^{3 / 2} T_{0}^{3 / 2}+\frac{4}{3} a T_{0}^{3}\right) \delta v_{0}
$$

In accordance with our hypothesis of irreversibility, however, we must expect

${ }^{28}$ See Eqs. (12) and (13), reference 7. 
the entropy of any given element of the fluid to increase ultimately without limit. Hence we can conclude from (38) that in a long series of expansions and contractions, the proper temperature and thus also the proper energy of any given element will be greater and greater at times when the volume of the element and "radius" of the model return to their earlier values; and vice versa that the volume and "radius" will become greater when the temperature returns to its earlier value. This is in agreement with our more general considerations in the preceding section.

\section{§. ConCLUSION}

The main purpose of this article has been a further examination of the bearings of relativistic thermodynamics on the well known problem of the entropy of the universe as a whole. The work has again illustrated the necessity of using relativistic rather than classical thermodynamics in treating this problem, and has demonstrated that the framework of general relativity at least provides a class of conceivable models of the universe which would undergo a continued series of expansions and contractions without being brought to rest by the irreversible processes which accompany these changes. The findings of relativistic thermodynamics thus stand in sharp contrast to the familiar conclusion of the classical thermodynamics that the continued occurrence of irreversible processes would lead to an ultimate condition of maximum entropy and minimum free energy where change would cease.

Attention has also been given to a somewhat special non-static model of the universe containing a uniform distribution of matter and radiation which by hypothesis could be converted one into the other. Such a model would undergo a succession of expansions and contractions and would provide possibilities in the later stages of expansion for the irreversible transformation of matter into radiation, and in the later stages of contraction for the irreversible transformation of radiation back into matter, without ever being brought to rest by thermodynamic obstacles. The result is of interest in connection with the annihilation or transformation of matter into radiation which the astrophysicists believe to be taking place in the actual universe.

It should perhaps be emphasized again, however, as I have previously done, that at present we discuss only very highly idealized models of the universe, partly on account of mathematical difficulties, and partly on account of the limited range of our observational knowledge which at best extends to some $10^{8}$ light-years. For example the hypothesis of roughly uniform conditions throughout the whole universe at a given time, which is assumed in the derivation of the non-static line element for the universe, can be justified only on the grounds that it introduces simplification into the mathematics, and that we have at present no observational knowledge to the contrary. For reasons such as these we must not be too dogmatic in our assertions as to the actual universe. The important thing at the present time is to investigate the properties of the highly simple and abstract models that we can treat, in order to gain ideas as to the general kind of phenomena that could conceivably be present in the actual universe. 